\title{
Hierarchical Combination of Unification Algorithms (Extended Abstract)
}

\author{
Serdar Erbatur ${ }^{5 *}$, Deepak Kapur ${ }^{1} \dagger$, Andrew M Marshall ${ }^{2} \ddagger$ Paliath Narendran ${ }^{3} \S$ \\ and Christophe Ringeissen ${ }^{4}$ \\ 1 University of New Mexico (USA) \\ 2 Naval Research Laboratory (USA) \\ 3 University at Albany, SUNY (USA) \\ 4 LORIA - INRIA Nancy-Grand Est (France) \\ 5 Dipartimento di Informatica Università degli Studi di Verona (Italy)
}

\section{Introduction}

A critical question in unification theory is how to obtain a unification algorithm for the combination of non-disjoint equational theories when there exists unification algorithms for the constituent theories. The problem is known to be difficult and can easily be seen to be undecidable in the general case. Therefore, previous work has focused on identifying specific conditions and methods in which the problem is decidable. We continue the investigation in this paper, building on previous combination results, [2, 4, 7] and 6]. We are able to develop a novel approach to the non-disjoint combination problem. The approach is based on a new set of restrictions and combination method such that if the restrictions are satisfied the method produces an algorithm for the unification problem in the union of non-disjoint equational theories.

A full version of this paper [5] will appear in the proceedings of the 24th International Conference on Automated Deduction (CADE-24).

\section{Preliminaries}

We use the standard notation of equational unification [3] and term rewriting systems [1]. A $\Sigma$-rooted term is a term whose top symbol is in $\Sigma$. Let $\Sigma_{(1,2)}=\Sigma_{1} \cap \Sigma_{2}$. An alien subterm of a $\Sigma_{1} \backslash \Sigma_{(1,2)}$-rooted term $t$ (resp. $\Sigma_{2}$-rooted term) is a $\Sigma_{2}$-rooted subterm (resp. $\Sigma_{1} \backslash \Sigma_{(1,2)}$-rooted subterm) of $t$ such that all its superterms are $\Sigma_{1} \backslash \Sigma_{(1,2)}$-rooted (resp. $\Sigma_{2}$-rooted). A theory $E$ is subterm collapse-free if and only if for all terms $t$ it is not the case that $t=_{E} u$ where $u$ is a strict subterm of $t$. Given a signature $\Sigma^{\prime} \subseteq \Sigma,\left.\mathcal{S}\right|_{\Sigma^{\prime}}$ denotes the set of $\Sigma^{\prime}$-equations occurring in $\mathcal{S}$. A set of equations $\mathcal{S}$ is said to be in standard form over a signature $\Sigma$ if and only if every equation in $\mathcal{S}$ is of the form $x=$ ? $t$, where $x$ is a variable and $t$ is one of the following: (a) a variable different from $x$, (b) a constant, or (c) a term of depth 1 that contains no constants, i.e., function symbols applied to variables. It is not generally difficult to decompose equations of a given problem into simpler standard forms. A set of equations is said to be in dag-solved form (or $d$-solved form) if and only if they can be arranged as a list $x_{1}=? t_{1}, \ldots, x_{n}=? t_{n}$

\footnotetext{
* Supported by the Department of Computer Science of the University at Albany and then INRIA NancyGrand Est during a portion of this work.

${ }^{\dagger}$ Partially supported by the NSF grant CNS-0905222

¥Supported by an ASEE postdoctoral fellowship under contract to the NRL.

$\S$ Partially supported by the NSF grant CNS-0905286
} 
where (a) each left-hand side $x_{i}$ is a distinct variable, and (b) $\forall 1 \leq i \leq j \leq n: x_{i}$ does not occur in $t_{j}$. Each $x_{i}$ in this case is called a solved variable. A set of equations $S$ is said to be in $\Sigma$-solved form if and only if it is in standard form and $\left.S\right|_{\Sigma}$ is in dag-solved form. For a convergent rewrite system $R$ we define a constructor of $R$ to be a function symbol $f$ which does not appear at the root on the left-hand side of any rewrite rule of $R$. We define an inner constructor to be a constructor $f$ that satisfies the following additional restrictions: (i) $f$ does not appear on the left-hand side on any rule in $R$, (ii) $f$ does not appear as the root symbol on the right-hand side of any rule in $R$, and (iii) there are no function symbols below $f$ on the right-hand side of any rule in $R$.

\section{Combination Procedure}

We want to investigate conditions to build an $E_{1} \cup E_{2}$-unification algorithm by using two algorithms $A_{1}$ and $A_{2}$ solving two different kinds of $E_{1} \cup E_{2}$-unification problems, described below in Restriction 1 and Restriction 2 respectively. Consider an $E_{1} \cup E_{2}$-unification problem $\mathcal{P}$ in standard form. The steps of the combination procedure (see Figure 2) are as follows: (1) run $A_{1}$ on the $\Sigma_{1} \backslash \Sigma_{(1,2)}$-equations of $\mathcal{P}$; (2) run $A_{2}$ on the $\Sigma_{2}$-equations of the unification problem obtained at step (1); (3) collect the resulting problems that are in dag-solved form. As in disjoint combination 2, we perform as step (0) a variable identification in order to guess a priori all possible identifications of variables occurring in $\mathcal{P}$. For this approach to work we need to place some restrictions.

\subsection{Restrictions}

We present a less technical overview of the required restrictions (see [5] for more details). Consider two subterm collapse-free equational theories such that $\Sigma_{1} \cap \Sigma_{2}=\Sigma_{(1,2)} \neq \emptyset$. Fresh variables created by $A_{1}$ that could cause the need for reapplication of the rules of $A_{1}$ during the application of $A_{2}$ are called "Ping-Pong Variables" as it implies a back-and-forth mechanism between the algorithms.

Restriction 1. (on Algorithm $\left.A_{1}\right)$ Let $\mathcal{P}$ be a set of $\Sigma_{1} \backslash \Sigma_{(1,2)}$-equations. Algorithm $A_{1}$ computes a set of problems $\left\{\mathcal{Q}_{k}\right\}_{k \in K}$ such that

$$
\bigcup_{k \in K} C S U_{E_{1} \cup E_{2}}\left(\mathcal{Q}_{k}\right) \text { is a } C S U_{E_{1} \cup E_{2}}(\mathcal{P}) \text { and for each } k \in K \text { : }
$$

(i) $\mathcal{Q}_{k}$ consists of $\left(\Sigma_{1} \backslash \Sigma_{(1,2)}\right)$-equations and $\Sigma_{(1,2)}$-equations.

(ii) $\mathcal{Q}_{k}$ is in standard form and $\left(\Sigma_{1} \backslash \Sigma_{(1,2)}\right)$-solved form.

(iii) No fresh variable occurring in a nonvariable $\Sigma_{(1,2)}$-term in $\mathcal{Q}_{k}$ appears as solved in $\mathcal{Q}_{k}$.

(CSU stands for "complete set of unifiers".)

Note that $A_{1}$ is a special type of algorithm that returns a "partial" solution to an $E_{1} \cup E_{2^{-}}$ unification problem. $A_{1}$ is needed to solve some portion of the problem, namely the $\Sigma_{1} \backslash \Sigma_{(1,2)^{-}}$ pure, but a standard $E_{1}$-unification algorithm is not sufficient, even for $\Sigma_{1} \backslash \Sigma_{(1,2)}$-pure $E_{1} \cup E_{2}$ problems.

Example 3.1. Let $E_{1}:=\{h(a, x, y)=g(x * y), h(b, x, y)=g(y * x)\}$ and let $E_{2}$ be the commutative theory for $*$. Then, $h(a, a, z)=? h(b, a, b)$ is not solvable in $E_{1}$ but is in $E_{1} \cup E_{2}$. 
Restriction 1 ensures that completeness is not lost by ensuring that a $C S U$ for all the partial solutions is a $C S U$ for the original problem.

To explain Restriction 1(iii), let us note that $A_{1}$ may generate fresh $\Sigma_{(1,2)}$-equations, e.g. $z=? f(x, y)$ where $f \in \Sigma_{(1,2)}$, together with some $\Sigma_{1} \backslash \Sigma_{(1,2)}$-equations, e.g. $x={ }^{?} s, y=? t$. If $A_{2}$ later generates $x=?$ ? then this may lead to the reapplication of $A_{1}$, to solve $x={ }^{?} s, x=?$ t If $x$ and $y$ are from the initial set of variables, this problem can be discarded without loss of generality, since the variable identification performed initially generates another unification problem where $x$ and $y$ are already identified. The problem remains if $x$ or $y$ are fresh variables, "ping-pong variables". In order to avoid it, we introduce Restriction 1(iii), where only the occurrences of fresh variables are restricted.

Restriction 2. (on Algorithm $A_{2}$ )

Algorithm $A_{2}$ computes a finite complete set of 2-pure unifiers of 2-pure $E_{1} \cup E_{2}$-unification problems.

We restrict $A_{2}$ to compute only 2-pure substitutions. This is used to avoid possible reapplications of $A_{1}$ after $A_{2}$.

Restriction 3. (Errors)

(i) $A \Sigma_{1} \backslash \Sigma_{(1,2)}$-rooted term cannot be $E_{1} \cup E_{2}$-equal to a $\Sigma_{2}$-rooted term.

(ii) $E_{1} \cup E_{2}$ is subterm collapse-free.

Note that in the next section we give a modularity result for subterm collapse-freeness. That is, we give a non-empty family of theories for which subterm collapse-freeness is a modular property.

The failure rules associated with Restriction 3 are given in Figure 1 .

Conflict: If $s(\epsilon) \in \Sigma_{1} \backslash \Sigma_{(1,2)}$ and $t(\epsilon) \in \Sigma_{2}:\{v=? s, v=? t\} \cup P \longrightarrow$ Fail

Cycle: If $P$ contains a cycle: $P \longrightarrow$ Fail

Figure 1: Failure rules

It can be shown based on these restrictions that Algorithm $\mathfrak{C}$ is a complete unification algorithm for the combined theory. More precisely, if $A_{1}$ and $A_{2}$ are algorithms satisfying Restrictions 1 through 3 , then Algorithm $\mathfrak{C}$ is sound and complete, which also means that $E_{1} \cup E_{2}$-unification is finitary.

\section{A Class of Hierarchically Combinable Theories}

In this section we define a non-empty class of theories for which Restrictions 1 through 3 can be satisfied. We assume that $E_{1}$ and $E_{2}$ are subterm collapse-free. The class is then defined by the following additional properties.

1. Properties of $E_{1}$ :

$R_{1}$ is a left-linear, convergent term rewrite system corresponding to $E_{1}$.

2. Properties of $E_{2}$ :

$E_{2}$ is a linear, finite equational theory. 
Some of the steps below are non-deterministic, hence lead to computation paths. Throughout each path, the failure rules of Figure 1 are applied eagerly after each step. Let $\mathcal{V}=\operatorname{Var}(\mathcal{P})$.

Step 0: Variable Identification

Guess a partition on $\mathcal{V}$ and set variables in each subset equal to each other. This requires adding fresh equations of type $u={ }^{?} w$, where $u, w \in \mathcal{V}$ and $u$ and $w$ belong to the same subset in the partition. Let us denote the enumeration of the partitions of $\mathcal{V}$ as $\pi_{1}, \pi_{2}, \ldots, \pi_{m}$. Once a partition is selected, the unification problem $\mathcal{P}$ is modified by adding the fresh equations between variables to the unification problem. We denote the modified problems by $\mathcal{P}^{\pi_{i}}, 1 \leq i \leq m$.

Step 1: Run $A_{1}$

We apply $A_{1}$ to the $\Sigma_{1} \backslash \Sigma_{(1,2)}$-equations of $\mathcal{P}^{\pi_{i}}$. If $A_{1}$ fails for all $\pi_{i}$, report failure and stop. Otherwise, we now have a modified, by $A_{1}$, set of unification problems from $\mathcal{P}^{\pi_{i}}, \mathcal{P}_{1}^{\pi_{i}}, \ldots \mathcal{P}_{n}^{\pi_{i}}$ for $n \geq 1$, such that all the $\Sigma_{1} \backslash \Sigma_{(1,2)}$-equations in each $\mathcal{P}_{j}^{\pi_{i}}$, are in $\left(\Sigma_{1} \backslash \Sigma_{(1,2)}\right)$-solved form.

Step 2: Run $A_{2}$

Run Algorithm $A_{2}$ on the 2-pure equations of each $\mathcal{P}_{j}^{\pi_{i}}$. If $A_{2}$ equates variables in $\mathcal{V}$ not equated by $\pi_{i_{j}}$ discard that particular $\mathcal{P}_{j}^{\pi_{i}}$. If none of the remaining $\mathcal{P}_{1}^{\pi_{i}}, \ldots \mathcal{P}_{n}^{\pi_{i}}$ exit with success, return failure. Otherwise, return success.

Figure 2: $\mathfrak{C}$ : Unification Algorithm for the combined theory $E_{1} \cup E_{2}$.

3. Properties of the shared symbols:

If $f \in \Sigma_{(1,2)}$, then $f$ is an inner constructor of $R_{1}$.

If $f$ and $g$ are inner constructors of $R_{1}$, then $f$-rooted terms cannot be equated to $g$-rooted terms in $E_{2}$.

Example 4.1. The equational theory $\mathcal{E}_{A C}$ considered in [6] satisfies all the above properties. The axioms of $\mathcal{E}_{A C}$ are as follows:

$$
\begin{aligned}
\exp (\exp (x, y), z)=\exp (x, y \circledast z) & (1) \quad(x \circledast y) \circledast z=x \circledast(y \circledast z)(3) \\
\exp (x * y, z)=\exp (x, z) * \exp (y, z)(2) & x \circledast y=y \circledast x(4)
\end{aligned}
$$

Here, $\mathcal{E}_{A C}=E_{1} \cup E_{2}$ where $E_{1}=\{(1),(2)\}$ and $E_{2}=\{(3),(4)\}$.

\subsection{Satisfying Restriction 1}

The first and critical step to satisfying Restriction 1 is the construction of an inference system $\mathcal{G}$ such that the standard forms of leaves in the search tree generated by $\mathcal{G}$ are the $\Sigma_{1} \backslash \Sigma_{(1,2)^{-}}$ solved forms corresponding to a complete set of $E_{1} \cup E_{2}$-unifiers of the input problem, thus satisfying the majority of Restriction 1. In [5, we modify the general syntactic $E$-unification procedure $\mathcal{G}$ [3] , but we still have to identify interesting cases for which $\mathcal{G}$ can be turned into a terminating algorithm. For the particular case of $\mathcal{E}_{A C}$, an algorithm $A_{1}$ satisfying Restriction 1 can be constructed from scratch as in [6].

\section{2 $\quad$ Satisfying Restriction 2}

Let $t^{\phi}$ denote the standard 2-abstraction of $t$, which corresponds to the 2-pure term obtained from $t$ by replacing alien subterms of $t$ with fresh variables $\left(E_{1} \cup E_{2}\right.$-equal alien subterms are replaced by the same fresh variable). It can be shown if $s \longleftrightarrow E_{E_{1}} \cup E_{2} t$, then $s^{\phi}=E_{E_{2}} t^{\phi}$. Reasoning inductively it can further be shown for any 2-pure equation $s={ }^{?} t$ where $s \sigma={ }_{E_{1}} \cup E_{2}$ 
$t \sigma$, that $s \sigma^{\phi}={ }_{E_{2}} t \sigma^{\phi}$. This implies that $E_{2}$-unification is sound and complete for solving 2pure $E_{1} \cup E_{2}$-unification problems. Hence, an $E_{2}$-unification algorithm provides an algorithm $A_{2}$ satisfying Restriction 2 since it computes a complete set of 2-pure unifiers.

\subsection{Satisfying Restriction 3}

Using the fact that $E_{2}$ is subterm collapse-free and the fact that if $s \longleftrightarrow E_{1} \cup E_{2} t$ then $s^{\phi}={ }_{E_{2}} t^{\phi}$, it can be shown that a $\Sigma_{1} \backslash \Sigma_{(1,2)}$-rooted term cannot be $E_{1} \cup E_{2}$-equal to a $\Sigma_{2}$-rooted term. Lastly, it can be shown that for any term $t$ and any strict subterm $u$ of $t$, if $t \rightarrow_{R_{1}}^{+} t^{\prime}$, then $t^{\prime} \neq_{E_{2}} u$. This fact leads directly to the result that $E_{1} \cup E_{2}$ is subterm collapse-free.

As a final remark, it is important to note that our algorithm $\mathfrak{C}$ does not work in a symmetric way with respect to $E_{1}$ and $E_{2}$, contrary to combination algorithms where we assume that any $\left(\Sigma_{1} \cap \Sigma_{2}\right)$-equality is valid in $E_{1}$ if and only if it is valid in $E_{2}$ [2, 4, 7. Indeed, the algorithm $A_{1}$ partially solves the problem modulo $E_{1} \cup E_{2}$ (and not only modulo $E_{1}$ ). Then, $A_{2}$ is in charge of solving modulo $E_{2}$ the remaining part that was kept unsolved by $A_{1}$. Our restrictions allow us to get all the solved forms without any further pingponging between $A_{2}$ and $A_{1}$.

\section{References}

[1] Franz Baader and Tobias Nipkow. Term rewriting and all that. Cambridge University Press, New York, NY, USA, 1998.

[2] Franz Baader and Klaus U. Schulz. Unification in the union of disjoint equational theories: Combining decision procedures. Journal of Symbolic Computation, 21(2):211 - 243, 1996.

[3] Franz Baader and Wayne Snyder. Unification theory. In John Alan Robinson and Andrei Voronkov, editors, Handbook of Automated Reasoning, pages 445-532. Elsevier and MIT Press, 2001.

[4] Eric Domenjoud, Francis Klay, and Christophe Ringeissen. Combination techniques for non-disjoint equational theories. In International Conference on Automated Deduction, (CADE-12), volume 814 of $L N C S$, pages 267-281. 1994.

[5] Serdar Erbatur, Deepak Kapur, Andrew M. Marshall, Paliath Narendran, and Christophe Ringeissen. Hierarchical combination. In Proceedings of CADE-24, volume 7898 of LNAI, pages 249-266. Springer, 2013.

[6] Serdar Erbatur, Andrew M. Marshall, Deepak Kapur, and Paliath Narendran. Unification over distributive exponentiation (sub)theories. Journal of Automata, Languages and Combinatorics (JALC), 16(2-4):109-140, 2011.

[7] Christophe Ringeissen. Unification in a combination of equational theories with shared constants and its application to primal algebras. In The 1st International Conference on Logic Programming and Automated Reasoning, volume 624 of LNAI, pages 261-272. Springer, 1992. 